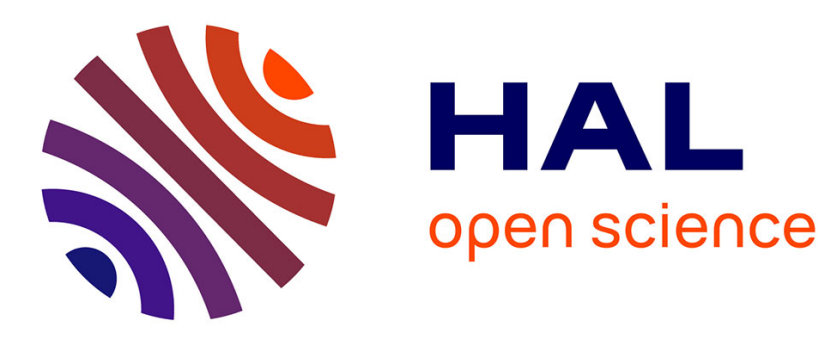

\title{
Impact of Connectivity Degradation on Networked Robotic Swarm Cooperation
}

Razanne Abu-Aisheh, Myriana Rifai, Francesco Bronzino, Thomas Watteyne

\section{To cite this version:}

Razanne Abu-Aisheh, Myriana Rifai, Francesco Bronzino, Thomas Watteyne. Impact of Connectivity Degradation on Networked Robotic Swarm Cooperation. IEEE International Conference on Distributed Computing in Sensor Systems (DCOSS), Jul 2021, Pafos, Greece. pp.57-59, 10.1109/DCOSS52077.2021.00021 . hal-03470202

\section{HAL Id: hal-03470202 \\ https://hal.science/hal-03470202}

Submitted on 8 Dec 2021

HAL is a multi-disciplinary open access archive for the deposit and dissemination of scientific research documents, whether they are published or not. The documents may come from teaching and research institutions in France or abroad, or from public or private research centers.
L'archive ouverte pluridisciplinaire HAL, est destinée au dépôt et à la diffusion de documents scientifiques de niveau recherche, publiés ou non, émanant des établissements d'enseignement et de recherche français ou étrangers, des laboratoires publics ou privés. 


\title{
(POSTER) Impact of Connectivity Degradation on Networked Robotic Swarm Cooperation
}

\author{
Razanne Abu-Aisheh ${ }^{* \dagger}$, Myriana Rifai*, Francesco Bronzino $^{\ddagger}$, Thomas Watteyne ${ }^{\dagger}$ \\ * Nokia Bell Labs, Paris, France \\ $\dagger$ Inria, EVA team, Paris, France \\ $\ddagger$ Université Savoie Mont Blanc, Annecy, France
}

\begin{abstract}
One of the most fundamental capabilities of swarm robotics is their ability to cooperate. This implies that swarm robots must exchange information with each other or with a centralized controller. However, this communication is often assumed to be perfect, an assumption that does not reflect real-world conditions, where impairments can affect the Packet Delivery Ratio (PDR) over wireless links. One essential application of swarm robotic cooperation is exploration and mapping in a timely and accurate manner. This paper studies how communication impairments can have a drastic impact on the performance of robotic swarms in critical missions such as exploration. We use an improved version of the Atlas algorithm to simulate the effect of various PDRs on the exploration mission execution performance, with the key indicator being mapping completion time. Our results show that the time it takes to complete area exploration increases exponentially as the PDR decreases linearly. Based on our results, we emphasise the importance of considering methods that minimize the delay caused by lossy communication when designing and implementing algorithms for robotic swarm exploration.
\end{abstract}

Index Terms-Swarm, Exploration, Mapping, Packet Loss, Micro-Robots.

\section{INTRODUCTION}

One of the essential applications of robotic swarm cooperation is the exploration of hazardous environments which is a risky, time and resource consuming mission. Autonomous swarm robotic exploration of dangerous environments has proven to be tremendously beneficial in terms of minimizing exploration time and reducing human exposure to risks [1]. A swarm of robots is defined as a group of at least three robotic entities that cooperate together to achieve a common global goal with limited to zero human operated control. During these operations, robots strategically search the environment to collect the most informative data from their surroundings. Thus, the ability to leverage the collected data to the benefit of other members of the swarm is critical to optimize the performance of the robots (e.g. minimize search completion time).

A typical approach to environment exploration consists of reaching full coverage of the explorable space - i.e. to map the environment - and leave no accessible parts of the environment unexplored [2] in the shortest time possible. Multiple Previous works have strongly focused on minimizing mission completion time due to the urgency and danger of typical search and rescue missions. However, many of these works still assume ideal communications between all members of the swarm, not

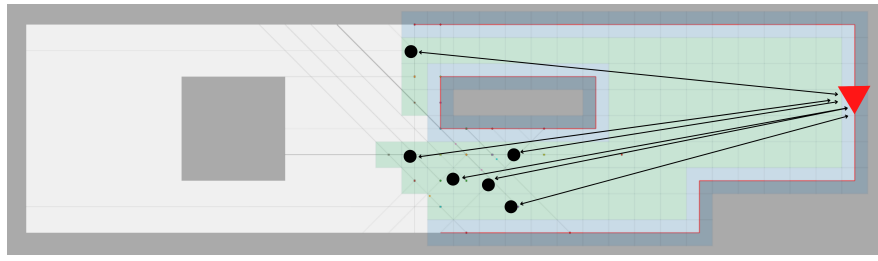

Fig. 1. The orchestrator and robotic swarm in an environment, showing a partially built map of a previously unknown environment.

taking into account communication limitations such as packet losses.

To evaluate the impact of network connectivity on mapping algorithms - and on robotic exploration based missions in general - this work studies the impact of lossy communication on the Atlas algorithm. We extend Atlas to take into account possible communication failures, while maintaining the guarantee of mapping completion. We develop and use a simplistic discrete-event continuous time simulator that includes more realistic communication conditions to evaluate the completion time even for extremely lossy environments. ${ }^{1}$.

This paper aims at demonstrating the importance of considering communication disturbances and losses when designing swarm cooperation algorithms for critical exploration based missions. The contributions of this paper are threefold:

- We develop a discrete-event, continuous-time and -space simulator that integrates lossy communication models.

- We design a modified version of the Atlas algorithm to include packet loss tolerant exploration and mapping that guarantees a $100 \%$ completion ratio.

- We emphasize the need for focusing on communication limitations when designing exploration algorithms by signifying the effect of packet loss on mission time-tocompletion.

\section{System Model And Problem Formation}

In this section, we give an overview of the system model elements and highlight the problems we address in our work.

\section{A. System Model}

1) Robots. We assume that each robot is proportionally small enough in comparison to the environment, where it can be

\footnotetext{
${ }^{1}$ As an online addition to this paper, the simulator is published under and open-source license at https://github.com/openwsn-berkeley/Atlas.
} 
modeled as a dot with $(\mathrm{x}, \mathrm{y})$ coordinates. We also assume that each robot can move at a speed of up to $1 \mathrm{~m} / \mathrm{s}$, has sensing capabilities limited to a bump sensor that is triggered upon contact with an obstacle, and antennas giving them communication capabilities.

2) Orchestrator. This central entity is responsible for the exploration strategy that robots should follow individually and collectively. The centralized nature of the orchestrator enables better exploration strategies based on its "global view" of the current state of the mission.

3) Environment and Communication. The environment is initially unknown to the system. All robots start the exploration from the location of the orchestrator and follow its instructions upon reception. The robots only report back to the controller when either of two possible events occur: a) when a robot's bump sensor is triggered, or b) when a robots assigned moving duration timer runs out (indicating that it has completed the movement instruction assigned by the orchestrator) In this manner, the swarm behaviour is asynchronous as the orchestrator updates the movement plan for that particular robot only when it hears back from it. We assume communication limitation by modelling packet loss into the environment in order to represent more realistic losses in a typical environment.

4) Exploration and Mapping. The exploration algorithm used is a packet-loss tolerant version of the Atlas algorithm from our previous work [3]. We refer to this version as "Atlas 2.0". The mapping is represented by dots with $(\mathrm{x}, \mathrm{y})$ coordinates on a continuous map, where each dot represents the location at which a robot's bump sensor was triggered. These dots connect into lines once they are a certain distance apart and create an outline of all the obstacles in an environment.

\section{B. Problem Formation}

In our work we focus on the main problem of developing and validating a mapping algorithm that reliably completes $100 \%$ of the time, within a reasonable amount of time, despite packet loss and communication impairments. This work led us to acknowledge the importance of factoring in the effects of communication degradation when designing algorithms that involve swarm robotic cooperation. We aim to demonstrate the impact of communication losses on cooperative swarm algorithms by showing the difference in performance of the same algorithm when assuming ideal communications vs. when taking packet losses into account. We evaluate the performance impact of communication losses in terms of the amount of time that takes to complete the mapping task.

\section{APPROACH}

\section{A. Communication Protocol}

To overcome the effect of communication impairments on mapping completion, we design a communication protocol that takes packet loss into account to guarantee mapping completion with any PDR above zero.

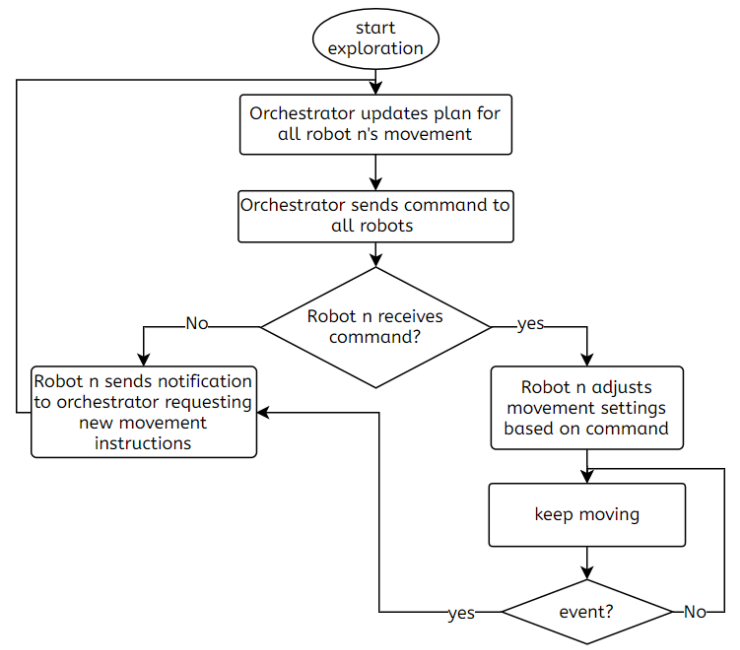

Fig. 2. A flow chart representing the communication between each robot and the orchestrator

As shown in Fig. 2, in our protocol, communication occurs between the orchestrator and each robot in the swarm (and vice-versa) through two types of packets: commands (from orchestrator to robot) and notifications (from robot to orchestrator). The commands contain the next movement instructions for all the robots; notifications contain the event that led the robot to stop, as well as the time stamps of the robots movements; one indicating when the robot started moving and another indicating when it stopped. This allows the orchestrator to keep track of accurate bump positions - and hence, obstacle locations - despite packet loss. This protocol is based on an event-based communication model with recurrent connectivity requirements: the robots only communicate back to the orchestrator once they have obtained new relevant data or have reached the assigned target position they were directed to go to. Otherwise, no connectivity is needed between the robots and the orchestrator.

\section{B. Exploration and Mapping}

In our previous work, we introduced Atlas: a centralized synchronous exploration algorithm specifically designed for sparse swarms [3]. It uses frontier-based systematic exploration; robots are controlled by a central orchestrator which maintains a partial map throughout the exploration and sends robots to explore the yet unexplored zones within the area. The frontier is the boundary between the explored area and that yet to be explored. The overall behavior is that the frontier expands "away" from the starting position: the robots are controlled to "push" the frontier further from the starting point. In scenarios where there are many obstacles, the swarm can be cut into subgroups as it navigates around obstacles. In that version of Atlas we assumed ideal lossless communications. In Atlas 2.0, we modify the previous version of Atlas to make it tolerant to lossy communications by incorporating the communication model mentioned in the previous section. When an event is triggered on the robots side, it keeps re- 
transmitting the notification until it receives a new command with new movement instructions. In addition, Atlas 2.0 is asynchronous: robots only get assigned new target destinations once they request them from the orchestrator. Otherwise, if no event occurs, the robots continue heading towards their assigned target destination, without the need for communicating with the orchestrator. While in the original version of Atlas, all robots would receive a new movement instruction every certain amount of time which would direct them to take one step further then all stop together, resulting in a collective dependency between robots.

In our system, the map represents an outline of the walls and obstacles in a bounded unknown environment, with the assumption that obstacles can be broken down into square shaped basic elements.

In our previous work [3], we introduce our open-source simulator that we developed specifically for comparing exploration and mapping algorithms. We build upon our previous work and improve the simulator for the purpose of investigating the effect of packet loss on event-based communication models. Added features include:

1) Continuous time and space

2) Asynchronous robotic movements

3) New mapping features

4) Improved User Interface

We assume all robots have "bump" sensors that get triggered whenever it hits an obstacle, as well as transceivers to enable communication between robots and the orchestrator.

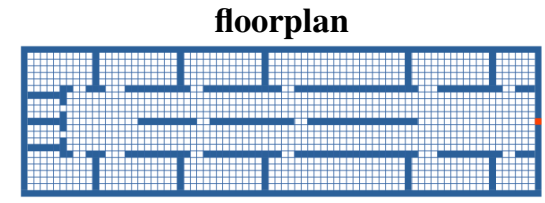

Fig. 3. The floorplan scenario used for the simulations with a size of $(80 \times 21$ cells $)$. The starting position is depicted as a red cell on the right.

\section{RESULTS AND DisCUSSION}

In this section, we detail how we ran our simulations and present and discuss the results obtained. Our aim is to emphasize the importance of considering more realistic communication assumptions while designing swarm robotic cooperation algorithms for critical missions by demonstrating the impact communication can have on mission time to completion.

In order to adequately demonstrate the impact of PDR, we run the simulations with various flat PDR rates across any point in the environment from 0.10 to 1.00 in steps of 0.10 . We run the simulations with a swarm of 50 robots. We run all cases with the floorplan scenario shown in Fig. 3. All results are presented with a $95 \%$ confidence interval.

Fig. 4 shows how the exploration time is impacted by the change in PDR. We can see that, as the PDR goes down, the exploration rate goes down with it and the time it takes to complete the mapping increases exponentially.

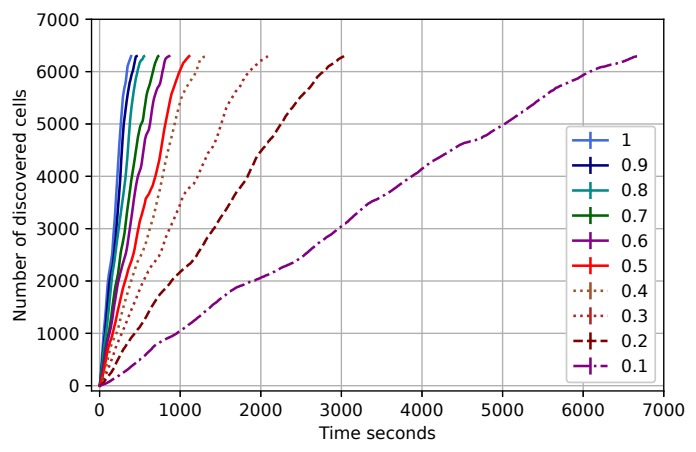

Fig. 4. Mapping profiles for different PDRs: the number of cells discovered over time for the floorplan scenario

In the floorplan use case, mapping completed in $6.5 \mathrm{~min}$ with no packet loss, 19 min with $50 \%$ packet loss, 1.85 hours with $90 \%$ packet loss. In critical missions such as search and rescue, hazard detection or chemical leakage, this delay in completing the mission could cost lives.

The conclusion we draw from our results is that the communication quality, the packet delivery ratio, and the communication protocol can have drastic impacts on the performance of swarm behaviour in critical missions requiring exploration. We therefore emphasize the importance of considering methods to compensate for the delay caused by lossy communication when designing and implementing algorithms for robotic swarm exploration.

\section{CONClusions AND FurTher WORK}

In this paper, we demonstrate the need for focusing on communication limitations when designing exploration algorithms by signifying the effect of packet loss on mapping time to completion. We run various simulations with various packet delivery ratios using an improved version of the Atlas algorithm on our discrete-event, continuous-time and -space opensource simulator that integrates lossy communication models. We show how, the higher the packet loss, the longer the mapping takes to complete. We therefore stress the importance of considering methods to compensate for the delay caused by lossy communication when designing and implementing algorithms for robotic swarm exploration. This research opens up several avenues for future work, including considering more realistic propagation models and taking into account scenarios where communication is completely lost.

\section{REFERENCES}

[1] B. Woosley, P. Dasgupta, J. G. Rogers, and J. Twigg, "Multi-Robot Information Driven Path Planning Under Communication Constraints," Autonomous Robots, vol. 44, no. 5, pp. 721-737, 2020.

[2] E. A. Jensen and M. Gini, "Effects of Communication Restriction on Online Multi-Robot Exploration in Bounded Environments," in Distributed Autonomous Robotic Systems. Springer, 2019, pp. 469-483.

[3] R. Abu-Aisheh, F. Bronzino, M. Rifai, B. Kilberg, K. Pister, and T. Watteyne, "Atlas: Exploration and Mapping with a Sparse Swarm of Networked IoT Robots," in 2020 16th International Conference on Distributed Computing in Sensor Systems (DCOSS). IEEE, 2020, pp. 338-342. 